\title{
Performance of hippocampectomized rats in a reference/working-memory task: Effects of preoperative versus postoperative training
}

\author{
P. D. GAGE \\ University of Maine, Orono, Maine
}

\begin{abstract}
Hippocampectomized and sham-operated male rats were pre- or postoperatively trained to perform a reference/working-memory task in a standard radial eight-arm maze. On each trial, three arms were baited. These "correct" arms formed one of two configurations, which changed between conditions. The remaining arms were never baited. The rats' task was to enter each of the baited arms only once, and to refrain from entering any of the unbaited arms. Incorrect choices were punished with a brief period of confinement. Preoperatively trained groups were tested postoperatively for retention, and then trained on a new reference-memory task in which the baited arms formed the second configuration. Postoperatively trained groups were also trained on two such tasks in succession. Order of training on the two configurations was counterbalanced between groups. Postoperatively trained hippocampal groups were severely impaired in acquisition of the tasks. Preoperatively trained hippocampal groups were unimpaired, relative to shamoperated groups, in all measures of performance.
\end{abstract}

The role of the hippocampus in processing spatial information is currently a topic of intensive investigation in the behavioral neurosciences. Much of the recent research in this area has been conducted to test various predictions following from the theories of O'Keefe and Nadel (1978) and of Olton, Becker, and Handelmann (1979).

According to O'Keefe and Nadel (1978), the hippocampus is the exclusive storage site of a nonegocentric representation of the spatial environment termed the cognitive map. This map encodes information regarding cues demarcating places, as well as the relative locations of these cues. Use of the cognitive map enables organisms to behave flexibly in complex spatial tasks; for example, they are capable of finding a particular location from any starting point, and of choosing efficient alternate routes when a familiar route is blocked. The "cognitive map" construct accounts for abundant evidence that normal rats tested in a familiar environment are capable of performing these spatial behaviors without specific training (e.g., Dashiell, 1930; Morris, 1981; O'Keefe, 1984, p. 384). In the O'Keefe and Nadel (1978) view, hippocampectomized organisms lack the capacity to form or utilize cog-

The author gratefully acknowledges the comments and suggestions of Merrill F. Elias, Michael Robbins, and Bill Thorton on earlier versions of this manuscript. Michael Abelson, Heather Wishart, Bill Prosser, Paul Welch, and Gerry Scarano provided assistance in building the apparatus, in histological analysis, and in running animals. Their contributions are deeply appreciated. Requests for reprints should be sent to P. D. Gage, Department of Psychology, University of Maine at Orono, Clarence Cook Little Hall, Orono, ME 04469. nitive maps, and therefore are severely impaired in the acquisition and retention of complex spatial tasks.

Olton et al. (1979) proposed that the hippocampus participates in working-memory processes to the exclusion of reference-memory processes. The former type of processes encode and retain information that is useful for a brief, specified duration (e.g., an experimental trial). On the other hand, reference-memory processes encode and retain information regarding the constants of an organism's environment (e.g., the information that remains useful throughout the duration of an experiment). In this view, hippocampectomized organisms should be differentially impaired in performing those aspects of a task requiring utilization of working-memory processes, regardless of whether they are pre- or postoperatively trained; on the other hand, acquisition and performance of reference-memory components of the task should be spared.

Acccording to a recently proposed alternative view, discussed in detail elsewhere (Gage, 1984, in press), the hippocampus plays a critical role in integrating and consolidating information for specific cognitive systems, including the spatial information that constitutes the cognitive map. During the process of cognitive mapping, this integrative function is specifically required to encode and store information regarding groups of cues demarcating places (O'Keefe \& Conway, 1978). Certain other types of learning capacity (e.g., associative learning) do not require this integrative function. For example, hippocampectomized rats can learn to approach a visible escape platform (cue task) in the Morris water maze task (Morris, Garrud, Rawlins, \& O’Keefe, 1982), but they are incap- 
able of learning the location of a hidden platform (place task). Unoperated animals acquire the latter task rapidly. It has been argued that the place task requires a functional cognitive map, whereas acquisition of the cue task is mediated by associative processes (e.g., Gage, in press; Sutherland, Kolb, \& Whishaw, 1982). Similar patterns of findings have been observed in other species and for other tasks (Gage, in press).

Furthermore, according to this view, the hippocampus is not a storage site for the cognitive map or for other information systems. Rather, the hippocampus participates in encoding the integrated information that constitutes these cognitive systems, and also plays a role in the consolidation of this information (cf. Squire, Cohen, \& Nadel, 1983). When the consolidation process is complete, hippocampal input is no longer required for maintenance of the cognitive map or other cognitive system. In this view, hippocampectomized rats are incapable of integrating and consolidating the complex spatial information required for the formation of a cognitive map. However, the preoperatively consolidated cognitive map remains functional following hippocampectomy. The literature concerned with amnesic effects of hippocampal damage in human and nonhuman primates provides evidence for such a dissociation (e.g., Squire et al., 1983). In these organisms, hippocampectomy causes a severe anterograde amnesia accompanied by a relatively mild partial retrograde amnesia. Furthermore, the human literature provides evidence that well-consolidated information is spared the amnesic effects of hippocampectomy. However, the available data provide equivocal evidence on the nature and extent of this dissociation in rats.

The purpose of the present experiment was to test the relative predictive value of these theories for the performance of hippocampectomized and sham-operated rats in a radial-maze task. The task was a variation of that developed by Olton and Papas (1979) for the purpose of dissociating tests of reference- and working-memory processes. In the present experiment, subjects were trained to retrieve food pellets concealed in recessed cups at the ends of each of three arms in an eight-arm maze. Any entry into a nonbaited arm was punished by confining the rat in the goal area of that arm. Reentries into correct arms after the bait had been retrieved (i.e., within a trial) were considered measures of working-memory error. Entries of arms that were members of the nonbaited set were considered reference-memory errors (Olton \& Papas, 1979).

The subjects were either preoperatively or postoperatively trained. Preoperatively trained hippocampal and sham-operated groups were tested postoperatively for retention of the preoperatively acquired task. Subsequently, they were trained on the same maze with a different set of baited arms. Postoperatively trained hippocampal and sham-operated groups were trained on the two maze tasks (i.e., on the two sets of arms) in succession.

The theories considered above make differential predictions for the performance of hippocampectomized rats in acquisition and retention of this task. The theory that the hippocampus is a storage site for cognitive maps (O'Keefe
\& Nadel, 1978) predicts that hippocampectomized organisms will be impaired in storage and retrieval of the nonegocentric spatial information required for performance of radial maze tasks (e.g., Suzuki, Augerinos, \& Black, 1980). According to this view, hippocampectomized rats should be impaired on all measures of acquisition and retention for this task.

The working-memory theory (Olton et al., 1979) predicts impairment of hippocampectomized subjects in all conditions (acquisition and retention, for both pre- and postoperatively trained groups), but only on the workingmemory components of these tasks. They should be unimpaired in the performance of reference-memory aspects of these tasks.

According to an alternative proposal (Gage, 1984), the hippocampus is critically involved in processing and consolidating spatial information. However, the preoperatively consolidated cognitive map is spared by hippocampectomy, and is accessible to mediate postoperative performance. On the other hand, postoperatively trained hippocampectomized rats should be severely impaired in measures of acquisition for this task.

The results are interpreted as consistent with the latter theory. The preoperatively trained hippocampal groups were unimpaired in retention tests for the preoperatively acquired task, and were also unimpaired in acquiring a new version of the task in the original experimental environment. Postoperatively trained hippocampal groups, however, were severely impaired in all measures of acquisition.

\section{METHOD}

\section{Subjects}

Subjects were 48 male Sprague-Dawley and SpragueDawley-derived rats, 6-9 months of age. The Sprague-Dawley rats were obtained from Charles River Breeding Laboratories. The Sprague-Dawley-derived rats were first- and second-generation descendants of Sprague-Dawleys bred in the animal laboratories at the Psychology Department, University of Maine at Orono. The 48 rats were each randomly assigned to one of the eight groups, which differed in the following conditions: (1) training condition (pre- or postoperative), (2) type of lesion (hippocampal or shamoperated), and (3) task sequence (two task sequences were counterbalanced). The experimental design is presented in Table 1.

The subjects had been maintained on ad-lib feed until they were 90 days of age. At that time they were placed on a deprivation schedule and reduced to $85 \%$ of their ad-lib weight. Thereafter they were weighed daily and were fed an amount of dry rat chow which maintained the $85 \%$ deprivation weight. During the experiment, the rats were always fed within a few minutes after being returned to their home cages.

\section{Surgery}

Animals were intraperitoneally injected with $75 \mathrm{mg} / \mathrm{kg}$ body weight of sodium pentobarbital (Nembutal), and were positioned in a Kopf or Scientific Instruments stereotaxic apparatus with the upper incisor bar and the interaural line in the same place. Trephine holes were drilled at eight sites along a single rostral-caudal plane. The coordinate for this plane was adjusted between 2.2 and $3.0 \mathrm{~mm}$ caudal to bregma, according to the weight of the animal. Medial-lateral coordinates within this plane were $\pm 4.5,3.2,2.2$, and $1.2 \mathrm{~mm}$ from the midline. All coordinates were determined on the basis of prior histological analyses of members of the same animal colony. 
Table 1

Experimental Design

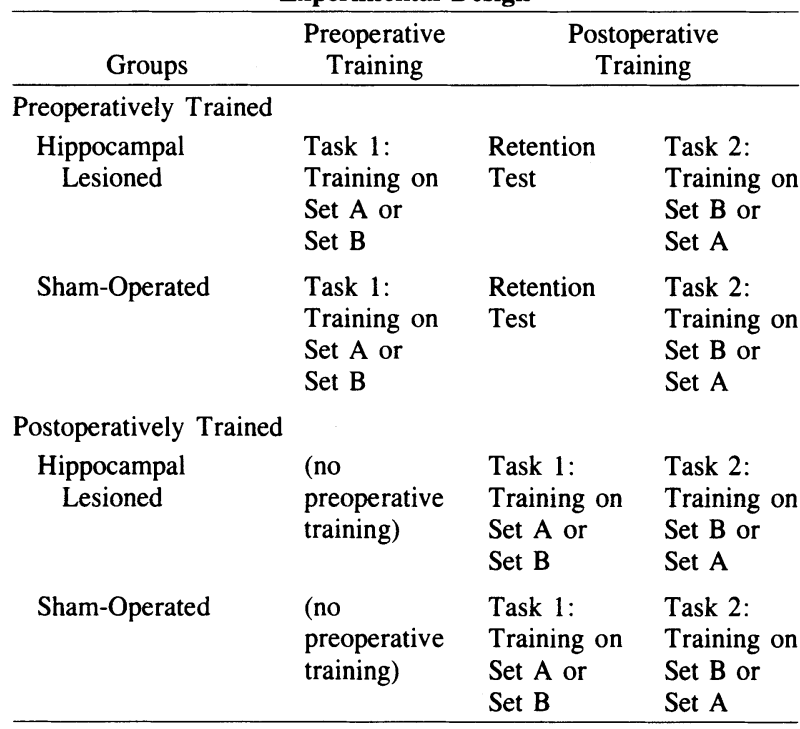

Note-Subjects trained on the Set A configuration in Task 1 were trained on the Set B configuration in Task 2; those trained on Set B in Task 1 were trained on Set $A$ in Task 2.

In animals scheduled for hippocampectomy, electrodes were inserted in the six most medial trephine holes to a depth of between 3.5 and $3.7 \mathrm{~mm}$ from the dura. The depth of the two most lateral insertions ( $1 \mathrm{~mm}$ lower than the medial insertions) was calculated to place the electrode tip within the lateral fimbria. Sham-operated rats underwent exactly the same surgical procedure as experimental animals, except that no electrodes were inserted.

Electrodes were made from size- 0 stainless steel insect pins that were coated with Electrolux insulation. The tips were scraped and ground under a dissecting microscope to $0.4 \mathrm{~mm}$. These electrodes served to pass anodal current into the brain; the cathodal electrode was placed into the anus. Lesions were made by passing $2 \mathrm{~mA}$ direct current for $20 \mathrm{sec}$ with an Applegate stimulator. Following completion of the hippocampal or sham surgery, animals were injected intramuscularly with $.05 \mathrm{ml}$ Combiotics.

Subjects were provided ad-lib food and water for 6 days postoperatively. Beginning on the 7 th postoperative day, they were handled for $5 \mathrm{~min}$ per day and were placed on the deprivation schedule described above. On the 13th postoperative day, pretraining trials commenced for postoperatively trained rats, and postoperative testing was begun for the preoperatively trained rats.

\section{Histology}

Within 3 days after a rat had completed running, he was heavily sedated with $150 \mathrm{mg} / \mathrm{kg} \mathrm{Nembutal}$ and was perfused through the heart with $10 \%$ formalin. Subsequently, the brain was removed and fixed in a $10 \%$ formalin solution. Brains were then embedded in a tissue-embedding medium and sliced at $11 \mu$ on a rotary microtome. Every fifth section was mounted, dewaxed, stained with Kluver-Bucy`s cresyl violet solution, and examined microscopically for the extent of tissue damage.

\section{Apparatus}

The apparatus was a modified version of the radial arm maze designed by Olton (Olton \& Samuelson, 1976), consisting of an octagonal center platform measuring $55 \mathrm{~cm}$ in diameter, and eight arms. each $91 \times 11 \mathrm{~cm}$, radiating from each side of the octagon. The maze was supported by posts at a height of $0.9 \mathrm{~m}$ from the floor. Each arm was bordered by a side wall $2.3 \mathrm{~cm}$ in height, except for a $7.2 \times 15.5-\mathrm{cm}$ guard near the center of the maze which prevented the rats from climbing across the arms. The maze floor and side walls were painted flat black. Located $1.5 \mathrm{~cm}$ from the end of the arms was a recessed food cup, $3 \mathrm{~cm}$ in diameter and $2.5 \mathrm{~cm}$ deep. At the entrance to each arm was a $10.1 \times 10.7 \mathrm{~cm}$ transparent Plexiglas guillotine door. These doors were termed inner doors. A second set of Plexiglas doors, termed outer doors, could be used to confine a rat at the goal end of each arm. These doors were located $26.5 \mathrm{~cm}$ from the ends of the arms. A Plexiglas panel was located above each door to prevent the rats from climbing over it. Guards of transparent soft Plexiglas were placed between the inner doors and also on either side of the outer doors; these guards prevented rats from climbing around the doors. The doors were controlled by a system of twine pulls threaded through overhead eyehooks to two separate manually operated control panels-one each for the outer and inner sets of doors.

The maze was located in a small $(2.9 \times 3.5 \mathrm{~m})$ experimental room containing several extramaze cues. The experimenter sat on a stool in one corner of the room, from where she or he operated the control panels and recorded data. The room was dimly lighted by a single overhead $40-\mathrm{W}$ incandescent bulb located behind a black shield.

\section{Procedure}

The sequence of conditions for preoperatively and postoperatively trained subject groups is summarized in Table 1.

All rats were handled daily, 2 min per day, for 2 weeks prior to their first scheduled pretraining trial (preoperatively trained groups) or prior to surgery (postoperatively trained groups).

Rats were given one trial per day, 6 days per week, at the same time each day. The maximum length of each trial was $10 \mathrm{~min}$.

During all trials, each arm entry was recorded. However, only entries to at least the arm's halfway point were defined as choices.

\section{Preoperatively Trained Groups}

Pretraining. Pretraining sessions proceeded in four stages: (1) Each rat was placed in the maze for at least one 10-min session until it moved freely about the maze. (2) Food pellets (Cocoa Puffs) were placed in each food cup, and the rat was allowed to retrieve the pellets. Rats were run on this and subsequent stages of pretraining until they made at least 8 choices within a trial. (3) Guillotine doors confined rats to the center of the maze at the beginning of the trial and following each choice. Confinement intervals lasted $5 \mathrm{sec}$ each. (4) The final stage of pretraining was conducted as previous pretraining sessions, except that rats were also confined for $5 \mathrm{sec}$ at the outer ends of the arms.

Task 1. Following completion of pretraining sessions, rats were trained on one of two reference/working-memory tasks, which differed only in the configuration of baited arms, referred to as Set A and Set B (see Figure 1). Rats were randomly assigned to these groups in counterbalanced fashion. For individual rats, the arms constituting the appropriate configuration were chosen from among those that were least often entered during pretraining trials.

Training proceeded in two stages. During the first stage, the rats were trained to enter the baited arms and to avoid the nonbaited arms. These trials were conducted as follows: Immediately prior to each trial, the correct arms were baited by placing three Cocoa Puffs in the recessed food cup of each correct arm. A trial began with the placement of the rat on the center platform with the inner doors closed. The rat was confined there for $10 \mathrm{sec}$, after which all doors were opened, allowing the rat to make a choice. After the rat entered the goal area of an arm, the outer doors were closed. If the choice was correct, the rat was confined only while he ate; if not. the rat was confined in the goal area for $12 \mathrm{sec}$. The outer doors were then opened, as was the inner door for that arm. Thus, the rat was allowed to return to the center of the maze, and was confined there for $7 \mathrm{sec}$ before being allowed to make another choice. A trial was terminated after the rat had retrieved food from the ends of the three correct arms, or after $10 \mathrm{~min}$ had passed, whichever occurred first.

This training procedure was continued for 12 days. During this procedure, errors were recorded and tabulated as either referenceor working-memory errors. Reference-memory errors were defined 


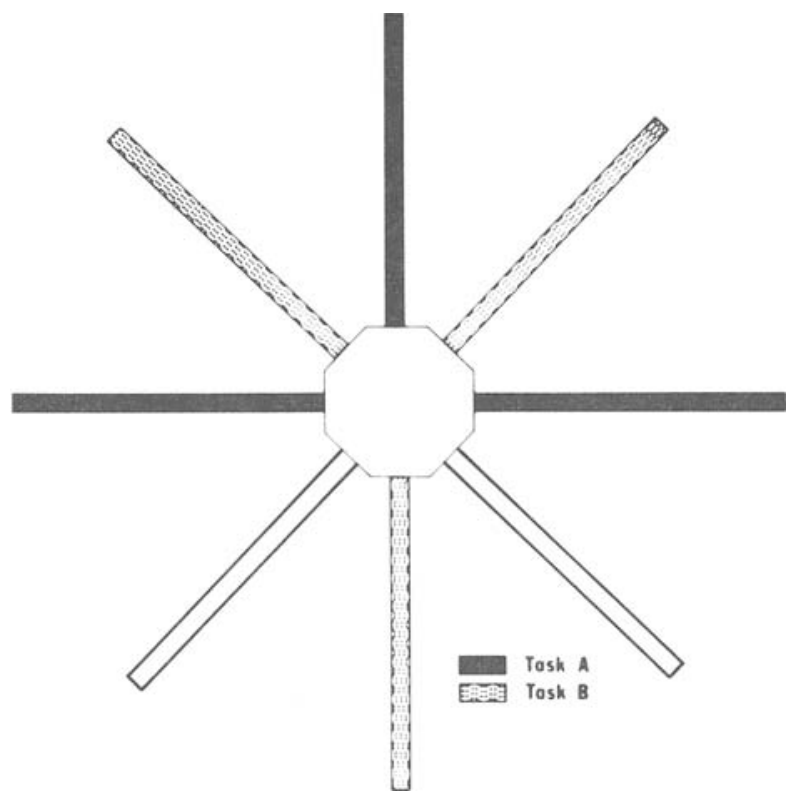

Figure 1. Graphic representation of the maze showing the configuration of baited arms in Set $A$ and Set $B$ conditions. In the actual maze, the arms were not differentially marked; rather, the entire maze was painted flat black.

as choices of arms that were not members of the baited set (Olton \& Papas, 1979); working-memory errors were defined as reentries into baited arms after the bait had been retrieved (Olton \& Papas, 1979).

On the day following completion of this procedure, the second stage of training was begun. During this stage, the rats were allowed only two errors per trial. If a rat made two errors (referencememory errors, working-memory errors, or one of each), he was removed from the maze for an approximately 24-h time-out. (In all other respects, the procedure was identical to that described above.) On the following day, the maze was baited as usual, and the rat was run on a new trial. This procedure was extensively tested in pilot studies. I found that rats trained with this procedure attained criterion more rapidly and with less variability than did subjects which continued with first-stage procedures. Within-subject performance was also more stable. An additional advantage of this procedure was that the reduced exposure to the maze during each trial resulted in a reduced opportunity for retraining during the retention test (described below).

Rats were tested until they reached a criterion of 7 consecutive days with no more than one error per day, and no more than four errors in total. The number of trials during the second stage of training required for the rats to reach this criterion was tabulated as the "trials-to-criterion"' dependent measure. Within 3 days after reaching criterion, rats underwent either hippocampal or sham surgery. Following the postoperative recovery period, these preoperatively trained rats were tested for retention.

Retention test. Following surgery, rats were run for 3 days on the first stage of the Task 1 training procedure described above. This retraining was continued until the rat had found all three members of the baited set of arms on 3 separate (not necessarily consecutive) days. As in Task 1, measures of reference- and workingmemory errors were obtained.

The second stage of the retention test began on the day following the third retention-test trial. The procedure was exactly as that described for the second stage of Task 1 training. Rats that did not reach criterion were terminated after 100 trials.
Following attainment of criterion during retention testing, the subjects were given a 2-day layoff. On these 2 days, rats were weighed and fed as described above, but were not run.

Task 2. Following layoff, rats which had been preoperatively trained on the Set A configuration of maze arms were now trained on Set B, and vice versa. Note that for any set of arms forming the Set A configuration, there was only one nonoverlapping Set B (see Figure 1). Thus, the individual arms that were members of the Task 2 configuration always differed from those that were members during Task 1 .

Procedures used during the first and second stages of Task 2 training were identical to those used in the first task. If a rat failed to reach criterion, testing was terminated after 100 trials.

\section{Postoperatively Trained Groups}

The sequence of conditions for postoperatively trained groups is specified in Table 1. These groups received no preoperative exposure to the maze. Following surgery and postoperative recovery, naive rats underwent pretraining sessions and then were trained on Task 1 according to the procedures detailed above. If a rat failed to reach criterion, training was terminated after 100 trials.

Only rats that attained criterion in less than 100 trials were trained on Task 2. After a rat reached criterion, he was given a 2-day
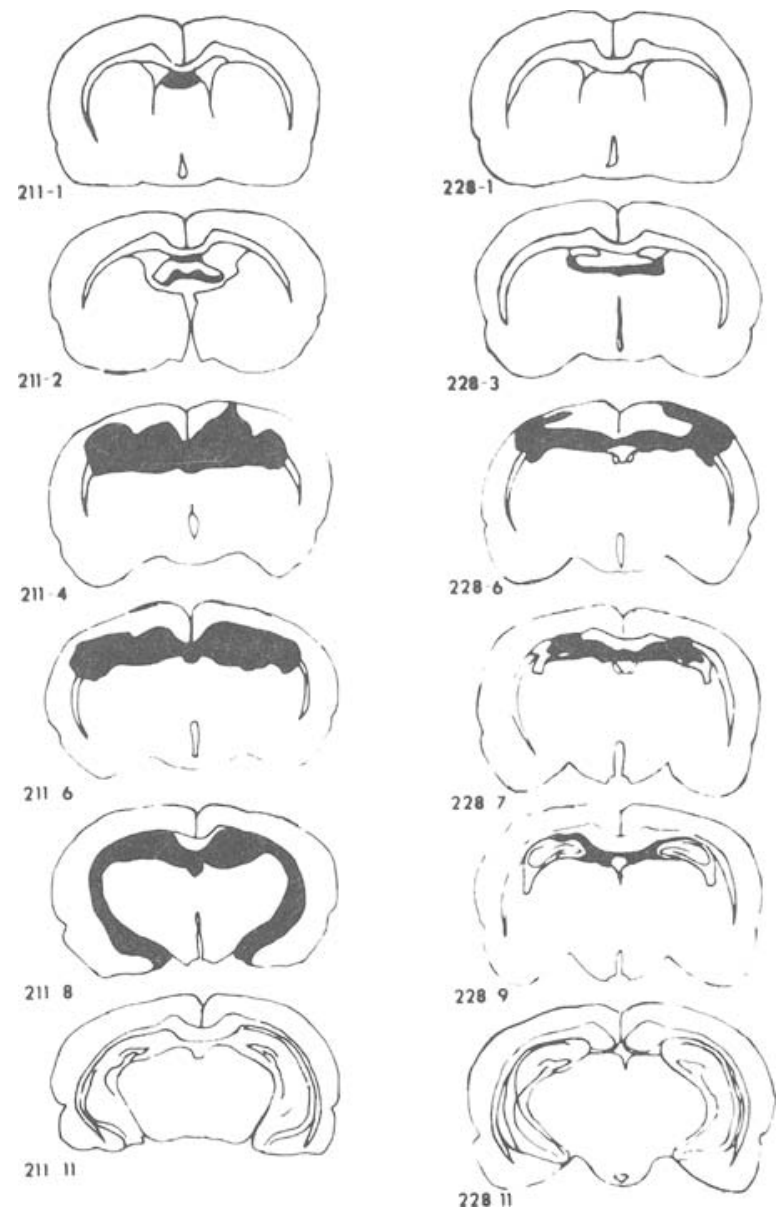

Figure 2. Tracings of sections obtained from the preoperatively trained hippocampectomized rats having the largest (left) and smallest (right) lesions. Labels refer to the animal identification numbers, followed by the number of the slide from which each section was traced. 
layoff, during which he was weighed and fed as usual, but was not run. Training and testing on Task 2 then proceeded in the manner detailed for preoperatively trained groups.

\section{RESULTS}

\section{Histological Results}

Serial reconstructions of the largest and smallest lesions in the pre- and postoperatively trained, hippocampal groups are represented in Figures 2 and 3. The surgical procedure was successful in accurately lesioning the dorsal hippocampus and lateral fimbria. In many animals, damage extended into the anteroventral hippocampus, hippocampal commissure, dorsal thalamic nucleus, and lateral caudate nuclei. In a few subjects ( 2 preoperatively trained and 1 postoperatively trained), gliosis of the rostral amygdala was observed. There was no discernible relationship between size of the lesion or particular extrahippocampal structures lesioned, and extent of the behavioral deficit.
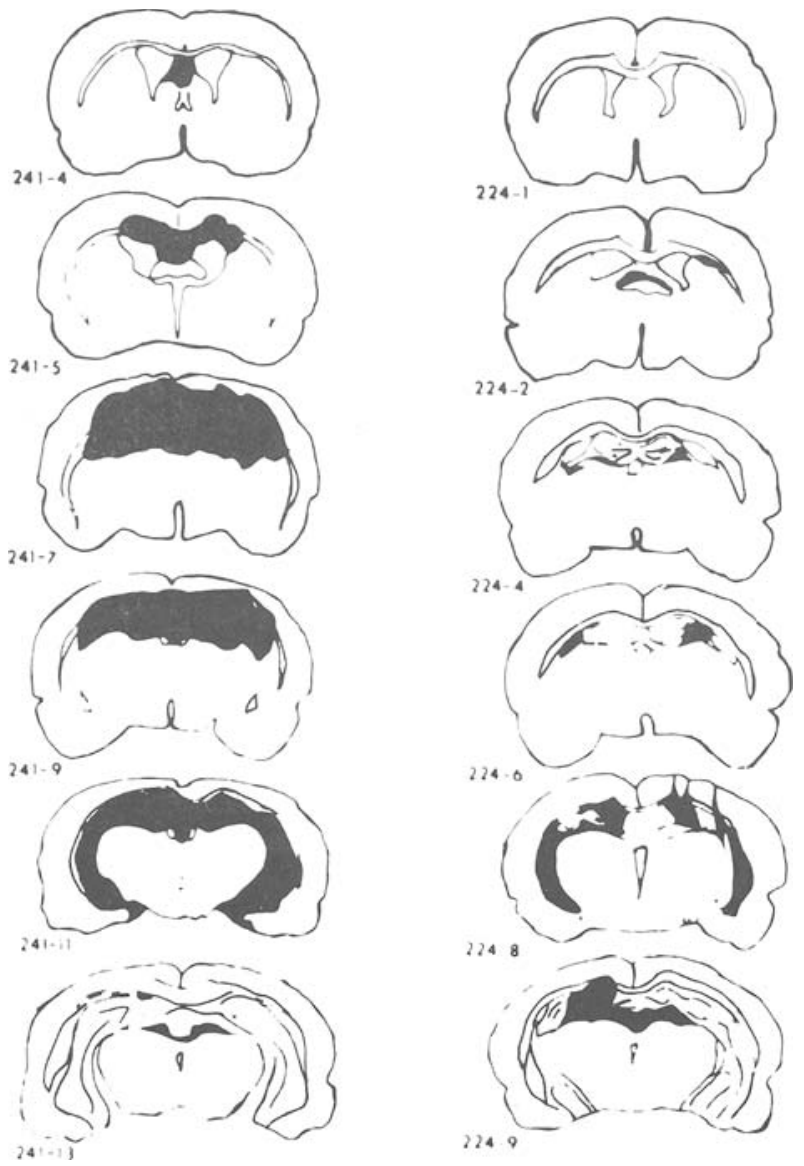

Figure 3. Tracings of sections obtained from the postoperatively trained hippocampectomized rats having the largest (left) and smallest (right) lesions. Labels refer to the animal identification numbers, followed by the number of the slide from which each section was traced.
Table 2

Mean ( \pm SE) Scores for Performance of Preoperatively Trained Hippocampal and Sham-Operated Groups on the Three Dependent Measures

\begin{tabular}{lrrrrrr}
\hline & & \multicolumn{2}{c}{ Task 1 } & \multicolumn{2}{c}{ Retention } & \multicolumn{2}{c}{ Task 2 } \\
\hline Trials to & $\mathrm{H}$ & $16.5 \pm 3.03$ & $12.3 \pm 1.83$ & $16.9 \pm 5.00$ \\
Criterion & $\mathrm{S}$ & $14.4 \pm 1.79$ & $11.1 \pm 2.76$ & $15.2 \pm 3.32$ \\
Reference- & $\mathrm{H}$ & $3.2 \pm .26$ & $3.3 \pm .83$ & $3.7 \pm$ & .36 \\
Memory Errors & $\mathrm{S}$ & $3.1 \pm .19$ & $1.3 \pm$ & \pm 58 & $2.8 \pm$ & .22 \\
Working- & $\mathrm{H}$ & $.3 \pm .08$ & $.5 \pm$ & \pm 21 & $.1 \pm$ & .03 \\
Memory Errors & $\mathrm{S}$ & $.2 \pm .05$ & $.2 \pm$ & \pm .21 & $.02 \pm$ & .01 \\
\hline
\end{tabular}

Note $-H=$ hippocampal group; $S=$ sham-operated group. $n=11$ for each group. Each dependent measure was obtained for each subject. The trials-to-criterion measures are group means. Group mean errors per trial are provided as measures of reference-and working-memory error.

\section{Behavioral Results}

Multivariate analyses of variance for repeated measures were conducted with type of lesion (hippocampal vs. sham) as the between-groups factor, and condition (Task 1, retention, Task 2) as the within-groups factor. The dependent measures of trials-to-criterion, referencememory errors, and working-memory errors have been defined in the Procedure section. The latter two measures were collected during the first stage of training of each condition. However, as described in the Procedure section, the number of trials in this stage varied between retention and task conditions. Therefore, the subjects' mean per-trial error rates were analyzed.

Preliminary analyses of the effects of task order (Set A prior to Set $B$, or vice versa) were nonsignificant in all cases. Therefore, the two levels of order, A-B and B-A, were combined for further analyses.

A major purpose of multivariate analysis of variance is to protect against the increase of experimentwise alpha error that occurs when several dependent variables are analyzed simultaneously (McCall \& Appelbaum, 1973). Therefore, in the following sections, univariate results are reported only when the Pillais multivariate-F statistic for that measure was significant.

Preoperatively trained groups. Two animals (1 preoperatively trained hippocampectomized rat and 1 preoperatively trained sham-operated rat) became seriously ill during training and were culled; their data were not included in the following analyses. Mean scores \pm standard errors for the remaining preoperatively trained subjects on the dependent measures are presented in Table 2.

Multivariate analyses of variance for the effects of the lesion factor revealed that the groups did not differ significantly on the performance measures $(p>.10)$. The lesion $\times$ condition interaction was also nonsignificant ( $p>$.10). However, the multivariate-condition main effect was significant $[F(6,15)=6.64, p<.001]$.

Analyses of contrasts were conducted for the effects of condition on each of the three dependent measures. Two contrasts were examined: (1) performance during the 
Task 1 versus retention-test conditions, and (2) performance during the Task 1 versus Task 2 conditions. These analyses revealed that both groups made significantly fewer working-memory errors in the Task 2 than in the Task 1 condition $[F(1,20)=6.35, p<.05]$. All other comparisons were nonsignificant.

Postoperatively trained groups. Three of the 12 postoperatively trained hippocampectomized animals (25\%) failed to attain crierion on Task 1 within 100 trials. None of the control subjects $(0 \%)$ failed to attain criterion within this or any other condition. The 3 rats which failed to attain criterion were not subjected to further training. Therefore, these subjects' data were not included in the overall analyses of repeated measures.

The data for the postoperatively trained groups are summarized in Table 3. Perusal of these data suggests that, compared with the sham-operated group, the hippocampal group was severely impaired in all measures of acquisition. Furthermore, the effects of the lesion were more severe in Task 1 than in Task 2. The multivariate analyses of variance corroborated these observations. The obtained multivariate-F statistics were significant for the lesion $[\mathrm{F}(3,17)=12.65, \mathrm{p}<.0005]$ and condition $[F(3,17)=14.79, p<.0005]$ main effects, and for the lesion $\times$ condition interaction $[\mathrm{F}(3,17)=5.57, \mathrm{p}<.01]$.

Univariate analyses revealed that the lesion $\times$ condition interaction was significant for the trials-to-criterion and the working-memory measures $[\mathrm{F}(1,19)=7.18$, $\mathrm{p}<.05 ; \mathrm{F}(1,19)=7.95, \mathrm{p}<.05$, respectively]. However, the lesion $\times$ condition interaction was not significant for reference-memory errors $(F=.00)$. The condition main effect was also nonsignificant for the reference-memory measure. However, perusal of Table 3 suggests that hippocampal groups were impaired in performance of reference-memory task components during both Task 1 and Task 2 . A significant lesion main effect $[\mathrm{F}(1,19)=26.31]$ corroborated this observation.

In order to clarify the interactions, analyses of simple main effects were conducted at each level of condition (i.e., Task 1 and Task 2) for trials-to-criterion and

Table 3

Mean ( \pm SE) Scores for Performance of Postoperatively Trained Hippocampal and Sham-Operated Groups on the Three Dependent Measures

\begin{tabular}{lrrrr}
\hline & & \multicolumn{2}{c}{ Task 1 } & \multicolumn{1}{c}{ Task 2 } \\
\hline Trials to & $\mathrm{H}$ & $57.7 \pm 10.41$ & $29.3 \pm 4.63$ \\
Criterion & $\mathrm{S}$ & $12.8 \pm 1.69$ & $10.8 \pm 1.69$ \\
Reference- & $\mathrm{H}$ & $5.0 \pm .41$ & $4.3 \pm .26$ \\
Memory Errors & $\mathrm{S}$ & $2.9 \pm .29$ & $2.8 \pm .16$ \\
Working- & $\mathrm{H}$ & $.9 \pm .17$ & $.3 \pm .12$ \\
Memory Errors & $\mathrm{S}$ & $.2 \pm .06$ & $.1 \pm .02$ \\
\hline
\end{tabular}

Note $-H=$ hippocampal group; $S=$ sham-operated group. During Task $1, n=12$ for both groups. However, 3 members of the hippocampal group did not reach criterion during Task 1, and were therefore not trained on Task 2. Each dependent measure was obtained for each subject. The trials-to-criterion measures are group means. Group mean errors per trial are provided as measures of reference- and workingmemory error.
Table 4

Magnitude of Lesion Effect as Measured by Adjusted $\mathbf{R}^{2}$

\begin{tabular}{lcc}
\hline & Task 1 & Task 2 \\
\hline Trials to Criterion & $.42 *$ & .13 \\
Reference-Memory Errors & $.42^{*}$ & $.60 *$ \\
Working-Memory Errors & $.38 \dagger$ & $.21 \ddagger$ \\
\hline
\end{tabular}

${ }^{*} p<.0005 . \quad \dagger p<.005 . \quad \ddagger p<.05$.

working-memory measures. Within Task 1 , the hippocampal and sham-operated groups differed significantly on these measures $[F(1,19)=17.99, p<.0005 ; F(1,19)$ $=15.34, \mathrm{p}<.001$; trials-to-criterion and workingmemory errors, respectively]. Within Task 2, the lesion simple main effect was significant for the workingmemory measure $[F(1,19)=6.32, p<.05]$, but not for the trials-to-criterion measure $[\mathrm{F}(1,19)=3.96, \mathrm{p}<.05]$. Thus, despite the presence of the interaction for the working-memory measure, lesioned rats were impaired relative to controls during training on both Task 1 and Task 2.

The matter of which dependent measure was most severely affected by the lesion was considered to be of theoretical interest. For example, Olton's (Olton et al., 1979) hypothesis predicts that hippocampal groups will be more severely impaired in the working-memory measure than in the reference-memory measure. The magnitude of $F$ values or relative probability levels of obtained statistics are inappropriate for evaluating this (relative magnitude of treatment effects) question (Keppel, 1982). Therefore, the conservative statistical test of the adjusted $\mathbf{R}^{2}$ (epsilon squared; Keppel, 1982) was conducted for the postoperatively trained groups. The statistic was not obtained for preoperatively trained groups because of the absence of significant effects of the lesion on performance for these groups (cf. Carroll \& Nordholm, 1975; Keppel, 1982).

The obtained $R^{2}$ statistics are summarized in Table 4 . During Task 1, the lesion was of approximately equal effectiveness in causing impairments on all measures. However, during Task 2, the reference-memory measure was severely affected by the lesion variable. The workingmemory measure was more mildly affected. The trialsto-criterion measure during Task 2 was not significantly affected by the lesion variable.

\section{DISCUSSION}

The findings reported here can be summarized as follows: Hippocampectomy resulted in a severe anterograde amnesia in naive, postoperatively trained rats. However, preoperatively trained hippocampectomized rats were unimpaired in retention; they showed no evidence of retrograde amnesia and no working-memory deficit. Furthermore, preoperatively trained hippocampal groups were unimpaired in acquiring Task 2 , even though they had not had specific preoperative experience in this task.

These results are inconsistent with O'Keefe and Nadel's (1978) view that the hippocampus is the storage site for 
cognitive maps. The pattern of results reported heresevere deficits observed in the postoperatively trained hippocampal groups along with unimpaired performance in preoperatively trained groups-suggests a role for the hippocampus in the processing (rather than storage) of spatial information.

Alternatively, it has been proposed that the hippocampus participates in integrative processes required for encoding and consolidating certain cognitive systems, including the cognitive map (Gage, 1984, in press). According to this view, the preoperatively consolidated cognitive map remains functional following hippocampectomy, but the organism's ability to construct a new cognitive map is severely impaired. The finding that preoperatively trained hippocampal groups did not suffer from retrograde amnesia is consistent with the hypothesis that a preoperatively acquired cognitive map was spared following hippocampectomy. The unimpaired performance of these hippocampal groups in acquiring Task 2 is also consistent with the view that hippocampectomy spares the preoperatively consolidated cognitive map: Successful performance of Task 2 required that the rats learn to approach new locations in a familiar environment. In this case, the familiar environment is represented by a preoperatively acquired cognitive map, which encodes an overall representation of the environment (i.e., the major landmarks and their relative spatial positions are encoded; O'Keefe \& Nadel, 1978; Suzuki et al., 1980). By this account, the cognitive map performs the same function in Task 2 for both the hippocampal and sham-operated groups: It mediates the rats' ability to approach new locations or to traverse new pathways in a familiar environment (e.g., Morris, 1981; O'Keefe \& Nadel, 1978; Roberts, 1984).

Of particular interest is the pattern of findings that, although preoperatively trained rats were unimpaired in acquisition, postoperatively trained rats suffered from a severe anterograde amnesia. These findings are consistent with the view that the hippocampus plays a critical role in processing information for the cognitive map.

The results reported here are inconsistent with Olton's hypothesis (e.g., Olton et al., 1979) that hippocampal lesions selectively impair working-memory functions. In the present experiment, preoperatively trained hippocampals were unimpaired in both measures of error, both during retention testing and during acquisition of Task 2 . Furthermore, performance on the working-memory measure improved between Task 1 and Task 2 for both the hippocampal and sham preoperatively trained groups.

On the other hand, postoperatively trained hippocampal groups were impaired in both measures of error. In fact, the lesion affected the performance of referencememory components of the task more severely than that of working-memory components, as evidenced by the adjusted $R^{2}$ statistics obtained for these groups (see Table 4).

The finding that postoperatively trained hippocampal groups are impaired in both reference- and workingmemory components of a complex spatial task is consis- tent with the literature. For example, Winocur (1982, Experiment 1) reported severe deficits in working-memory processes for postoperatively trained hippocampals. Deficits for postoperatively trained hippocampals in reference-memory tasks have also been demonstrated (Harley, 1979; Morris et al., 1982).

However, the literature concerned with the effects of hippocampectomy on performance of working-memory and reference-memory components of a preoperatively acquired task is equivocal. For example, Harley (1979) found that hippocampals were unimpaired in retention of a preoperatively acquired Tolmanian sunburst maze task (reference-memory task). Furthermore, Winocur (1982) found evidence of negative transfer in rats that were preoperatively trained on a noncued radial maze, then retrained postoperatively on a cued maze. No such impairment occurred in control groups trained in this sequence of conditions. Winocur's (1982) findings are evidence that preoperatively acquired spatial information is retained and interferes with postoperative learning.

On the other hand, Jarrard (1983; Jarrard \& Elmes, 1982) reported results that do not agree with the present findings. Jarrard used a task similar to that used here-a radial-maze task with explicit working-memory and reference-memory components-and conducted in a similar experimental environment. Preoperatively trained hippocampectomized subjects were severely impaired in measures of reference and working memory during postoperative retention tests. However, there were several methodological differences between the studies that might account for the discrepant results. For example, Jarrard performed aspiration lesions, whereas in the present study, electrolytic lesions were made at eight dorsal hippocampal sites. A precise comparison of the extent of the lesions in the two studies is not possible. However, the discrepant findings may be attributable to differences in the extent of the hippocampal lesions, to differences in the amount of neocortical tissue damage (which is greater in the Jarrard, 1983, and Jarrard \& Elmes, 1982, experiments than in the present experiment), or to other differential effects of the two techniques.

Another factor that may have contributed to the discrepant results is this study's procedure of punishing incorrect choices with a confinement or "time-out" period. It is reasonable to speculate that use of the time-out procedure in the present study may have differentially increased the hippocampal subjects' motivation to inhibit entries of incorrect arms. This suggestion is consistent with evidence that signals of nonreward are ineffective in controlling the behavior of hippocampectomized rats (e.g., Gray, 1982). However, hippocampectomized rats are capable of inhibiting a punished approach response (e.g., Cogan \& Reeves, 1979; Gage, in press). Thus, the deficit reported by Jarrard (1983; Jarrard \& Elmes, 1982) may be at tributable to a performance deficit (failure to inhibit the nonrewarded response) rather than to a retention deficit. However, further research is necessary to test this hypothesis. 


\section{REFERENCES}

Carroll, R. M., \& Nordholm, L. A. (1975). Sampling characteristics of Kelley's $\epsilon^{2}$ and Hay's $\hat{\omega}^{2}$. Educational \& Psychological Measurement, 35, 541-554.

Cogan, D. C., \& Reeves, J. L. (1979). Passive avoidance learning in hippocampectomized rats under different shock and intertrial interval conditions. Physiology \& Behavior, 22, 1115-1121.

DASHIELL, J. F. (1930). Direction orientation in maze running by the white rat. Comparative Psychology Monographs. 7, 1-72.

GAGE, P. D. (1984). Hippocampal mediation of complex spatial learning: A test of the differential predictions of three theories. Unpublished doctoral dissertation, University of Maine at Orono.

GAGE, P. D. (in press). Preserved and impaired information processing systems in human bitemporal amnesiacs and their infrahuman analogues: Role of hippocampectomy. Journal of Mind \& Behavior.

GraY, J. A. (1982). The neuropsychology of anxiety: An enquiry into the functions of the septo-hippocampal system. New York: Oxford Press.

Harley, C. W. (1979). Arm choices in a sunburst maze: Effects of hippocampectomy in the rat. Physiology \& Behavior, 23, 283-290.

JARRARD, L. E. (1983). Selective hippocampal lesions and behavior: Effects of kainic acid on performance of place and cue tasks. Behavioral Neuroscience, 97, 873-889.

JARRARD, L. E., \& ElMES, D. G. (1982). Role of retroactive interference in the spatial memory of normal rats and rats with hippocampal lesions. Journal of Comparative and Physiological Psychology, 96, 699-711.

KePPEL, G. (1982). Design and analysis: A researcher's handbook. Englewood Cliffs, NJ: Prentice-Hall.

McCall, R. B., \& Applebaum, M. I. (1973). Bias in the analysis of repeated measures designs: Some alternative approaches. Child Development, 44, 401-415.

MoRRIS, R. G. M. (1981). Spatial localization does not require presence of local cues. Learning \& Motivation, 12, 239-260.

Morris, R. G. M., Garrud, P., Rawlins, J. N. P., \& O'Keefe, J. (1982). Place navigation impaired in rats with hippocampal lesions. Nature, 297, 681-683.
O'KEEFE, J. (1984). Spatial memory within and without the hippocampal system. In W. Seifert (Ed.), Neurobiology of the hippocampus. New York: Academic Press.

O'Keefe, J., \& Conway, D. H. (1978). Hippocampal place units in the freely moving rat: Why they fire where they fire. Experimental Brain Research, 31, 573-590.

O'KeEFE, J., \& NADEL, L. (1978). The hippocampus as a cognitive map. Oxford: Oxford University Press.

Olton, D. S., Becker, J. T., \& Handelmann, G. E. (1979). Hippocampus, space, and memory. Behavioural \& Brain Sciences, 2, 313-365.

Olton, D. S., \& PaPAS, B. C. (1979). Spatial memory and hippocampal function. Neuropsychologia, 17, 669-682.

Olton, D. S., \& Samuelson, R. J. (1976). Remembrance of places passed: Spatial memory in rats. Journal of Experimental Psychology, 8, 289-314.

RoBerTs, W. A. (1984). Some issues in animal spatial memory. In H. L. Roitblat, T. G. Bever, \& H. S. Terrace (Eds.), Animal cognition. Hillsdale, NJ: Erlbaum.

Squire, L. R., Cohen, N. J., \& Nadel, L. (1983). The medial temporal region and memory consolidation: A new hypothesis. In H. Weingartner \& E. Parker (Eds.), Memory consolidation: Towards a psychobiology of cognition. Hillsdale, NJ: Erlbaum.

Sutherland, R. J., KolB, B., \& Whishaw, I. Q. (1982). Spatial mapping: Definitive disruption by hippocampal or medial frontal cortical damage in the rat. Neuroscience Letters, 31, 271-276.

Suzuki, S., Augerinos, G., \& BlaCK, A. H. (1980). Stimulus control of spatial behavior on the eight-arm radial maze in rats. Learning \& Motivation, 11, 1-18.

WINOCUR, G. (1982). Radial-arm-maze behavior by rats with dorsal hippocampal lesions: Effects of cuing. Journal of Comparative \& Physiological Psychology, 96, 155-169.

(Manuscript received November 2, 1984; revision accepted for publication October 25,1985 .) 